\title{
Congenital Muscular Dystrophy with Rigid Spine Syndrome: A Clinical, Pathological, Radiological, and Genetic Study
}

\author{
Kevin M. Flanigan, MD, ${ }^{*} \dagger \ddagger$ Lynne Kerr, MD, PhD, ${ }^{*} \$$ Mark B. Bromberg, MD, PhD, ${ }^{*}$ \\ Claire Leonard, MD, $\dagger \dagger$ Jay Tsuruda, MD, 9 Ping Zhang, MD, Ignacio Gonzalez-Gomez, MD,\# \\ Ronald Cohn, MD, ${ }^{* *}$ Kevin P. Campbell, PhD, ${ }^{* *}$ and Mark Leppert, $\mathrm{PhD}^{\|}$
}

\begin{abstract}
Rigid spine syndrome is a term first proposed by Dubowitz to describe a subset of patients affected by myopathy with early spinal contractures as a prominent feature. While spinal rigidity is a nonspecific feature, found in Emery-Dreifuss muscular dystrophy and in some congenital myopathies, it is also a prominent feature in a group of patients with merosin-positive congenital muscular dystrophy, where it is generally associated with stable or only slowly progressive weakness and early respiratory insufficiency. Recently, the first locus for congenital muscular dystrophy in association with rigid spine syndrome was mapped to chromosome 1p35-p36 in consanguineous Moroccan, Turkish, and Iranian families. We present here a detailed phenotypic description of the familial syndrome linked to this locus, describing 4 siblings ( 3 boys and 1 girl) of Northern European-American heritage who are the offspring of a nonconsanguineous marriage. All 4 siblings were affected by hypotonia and prominent neck weakness in infancy, early spinal rigidity, and early scoliosis. After initial improvement, muscle strength stabilizes or slowly declines, and skeletal deformities and respiratory insufficiency supervene. Muscle biopsy in an affected child at age 9 months revealed minimal, nonspecific myopathic changes, leading to a diagnosis of "minimal change myopathy." Muscle biopsy in his sibling, at the age of 14 years, revealed chronic and severe myopathic (dystrophic) changes, with normal staining for laminin-2 and for proteins of the dystrophin-glycoprotein complex. A possible explanation for these biopsy findings is that magnetic resonance imaging of the thighs reveals stereotyped selective muscle involvement, with the selectivity more pronounced early in the disease course followed by widespread muscular signal abnormalities in the late stages of the disease. In this family, linkage to the chromosome 1p rigid spine syndrome locus (RSMD1) is supported by maximum LOD scores for several markers of 1.81 at $\Theta=0$, representing the maximum statistical power possible for this family. In combination with the previous report, this syndrome is linked to the RSMD1 locus with a summated maximum LOD score of 6.29, and analysis of recombination events in our family narrows the previously reported RSMD1 locus to 3 centiMorgans.
\end{abstract}

Flanigan KM, Kerr L, Bromberg MB, Leonard C, Tsuruda J, Zhang P, Gonzalez-Gomez I, Cohn R, Campbell KP, Leppert M. Congenital muscular dystrophy with rigid spine syndrome: a clinical, pathological, radiological, and genetic study. Ann Neurol 2000;47:152-161

The congenital muscular dystrophies (CMDs) are a group of disorders marked by hypotonia at birth and a generally nonprogressive course of muscle weakness. ${ }^{1,2}$ A large proportion of cases are the result of a deficiency of the protein laminin-2, or merosin, ${ }^{3}$ resulting in the absence of that protein on immunohistochemical studies and associated with mutations in the LAMA2 gene. ${ }^{4}$ The merosin-positive CMDs constitute a genetically heterogeneous group. ${ }^{5}$ Some merosin-positive forms have striking central nervous system abnormalities, including Fukuyama CMD, caused by mutations in a gene on chromosome $9 \mathrm{q}$ encoding the novel protein fukutin, ${ }^{6}$ muscle-eye-brain disease, recently mapped to chromosome 1p32-p34, ${ }^{7}$ and Walker-Warburg CMD, which has some clinical similarities to muscle-eye-brain disease. In addition, a large proportion of patients do not have specific associated features. ${ }^{2,5}$ In some of these syndromes, particularly Fukuyama CMD and muscle-eye-brain disease, ${ }^{8}$ there may be a secondary reduction of merosin in muscle, but they may still be considered part of the merosin-positive category of CMD.
From the Departments of *Neurology, †Pathology, IRadiology, Human Genetics, and ††Pediatrics, and $\ddagger$ Program in Neuroscience, University of Utah, and $\$$ Department of Physical Medicine and Rehabilitation, Primary Children's Medical Center, Salt Lake City, UT; \#Department of Pathology, Children's Hospital, Los Angeles, CA; and ${ }^{* *}$ Department of Physiology and Biophysics, Department of Neurology, and Howard Hughes Medical Institute, University of Iowa, Iowa City, IA.
Received Jun 8, 1999, and in revised form Sep 23. Accepted for publication Sep 24, 1999.

Address correspondence to Dr Flanigan, Eccles Institute of Human Genetics, Room 2100, 15 N. 2030 East Street, Salt Lake City, UT 84112. 
Because a significant number of merosin-positive CMD patients have spinal rigidity as a prominent feature of the illness, a rigid spine syndrome (RSS) phenotype has been proposed as a subtype of CMD. ${ }^{5}$ The term rigid spine syndrome was first proposed by Dubowitz $^{9,10}$ in describing a condition resembling muscular dystrophy, with onset in infancy but a relatively benign course of muscle weakness, associated with early and severe spinal and limb joint contractures. Nocturnal hypoventilation may be an early feature and a cause of mortality. ${ }^{11,12}$ Spinal rigidity, however, is not a specific finding; it is a prominent feature in the X-linked, autosomal dominant, ${ }^{13}$ and autosomal recessive $^{14}$ forms of Emery-Dreifuss muscular dystrophy and in Bethlem myopathy, and has been reported in nemaline and other congenital myopathies. ${ }^{15,16}$ In addition, rigid spine syndrome has been used for a syndrome of late-onset myopathy with prominent spinal extensor contractures. ${ }^{17}$ Several authors have pointed out a distinction between spinal rigidity in other myopathies and a clinically distinct RSS, ${ }^{18}$ and some have cast doubt on the existence of a distinct syndrome. ${ }^{19}$ However, designation of a CMD-RSS subset has proven useful, as recently a first locus for this syndrome was identified on chromosome $1 \mathrm{p} .{ }^{20}$ This locus was found by the use of homozygosity mapping ${ }^{21}$ in a population of consanguineous Moroccan, Turkish, and Iranian patients; genetic heterogeneity for this syndrome was demonstrated by families not linked to this locus. Five patients from three families mapped to the locus displayed a moderately heterogeneous clinical picture, with variability in the distribution of weakness, degree of scoliosis, and severity of functional disability; all, however, had early respiratory insufficiency and spinal rigidity. ${ }^{20}$ The authors of that report termed this locus RSS, but that designation was already assigned to the locus for Russell-Silver Syndrome by the Human Gene Organization Nomenclature Committee. The designation RSMD1 (for rigid spine muscular dystrophy 1) has now been assigned to this locus by the Committee.

We report the clinical, pathological, and radiological characteristics of 4 siblings affected by CMD mapping to the chromosome $1 \mathrm{p} 35$ RSMD1 locus. Clinically, hypotonia and neck weakness are early features, followed by early scoliosis, muscle atrophy, and nocturnal respiratory insufficiency. Pathologically, myopathic changes on muscle biopsy vary from minimal, nonspecific change to severe, chronic myopathic (dystrophic) changes. Magnetic resonance imaging (MRI) of muscle reveals selective muscle involvement in early stages of the disease, suggesting that muscle selection by muscle MRI scanning may increase the diagnostic yield of a muscle biopsy early in the disease course. Genotyping of markers at the RSMD1 locus on chromosome $1 \mathrm{p}$ strongly suggests linkage, and haplotype analysis reveals obligate recombination events that narrow the RSMD1 gene region to approximately 3 centiMorgans.

\section{Patients and Methods}

\section{Patients}

The 4 children (Figs 1 and 2) were born after uncomplicated pregnancies. The parents are both of Scandinavian-American heritage, but their ancestors are reportedly from different regions of Scandinavia. More recent ancestors lived on adjacent farms in Nauvoo, Illinois, before emigration to Utah in the mid-19th century, but there is no known consanguinity. Neurological examination of both parents is entirely normal.

PATIENT 30357. The oldest child, Patient 30357 (see Figs 1 and 2D), is now 17 years old. He was noted to be floppy at birth, and to have undescended testicles and coarctation of the aorta (repaired by balloon angioplasty at age 10 years). At 6 months of age, he could sit unsupported, although he could not lift his arms above his head, and his tendon reflexes were present although symmetrically decreased. A head computed tomographic scan and an electroencephalogram were normal, and a serum creatine kinase was within the normal range. A muscle biopsy was performed in the right quadriceps at the age of 9 months and was interpreted as "minimal change myopathy" (see pathology section and Fig $3 \mathrm{~A}$ and $\mathrm{B})$. Although he had poor head control in the first years of life, his strength gradually improved; he walked by

Fig 1. Left to right: Patient 30360 (age 7 years); Patient 30359 (age 11 years); Patient 30358 (age 14 years); and Patient 30357 (age 17 years). Note decreased muscle bulk and scoliosis.

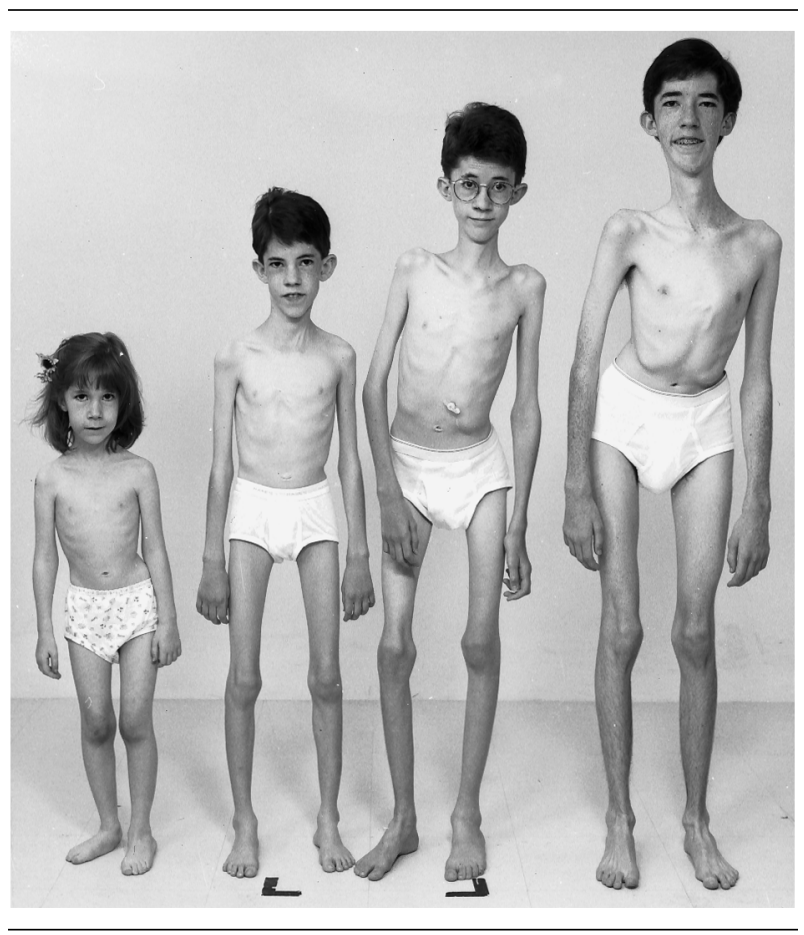



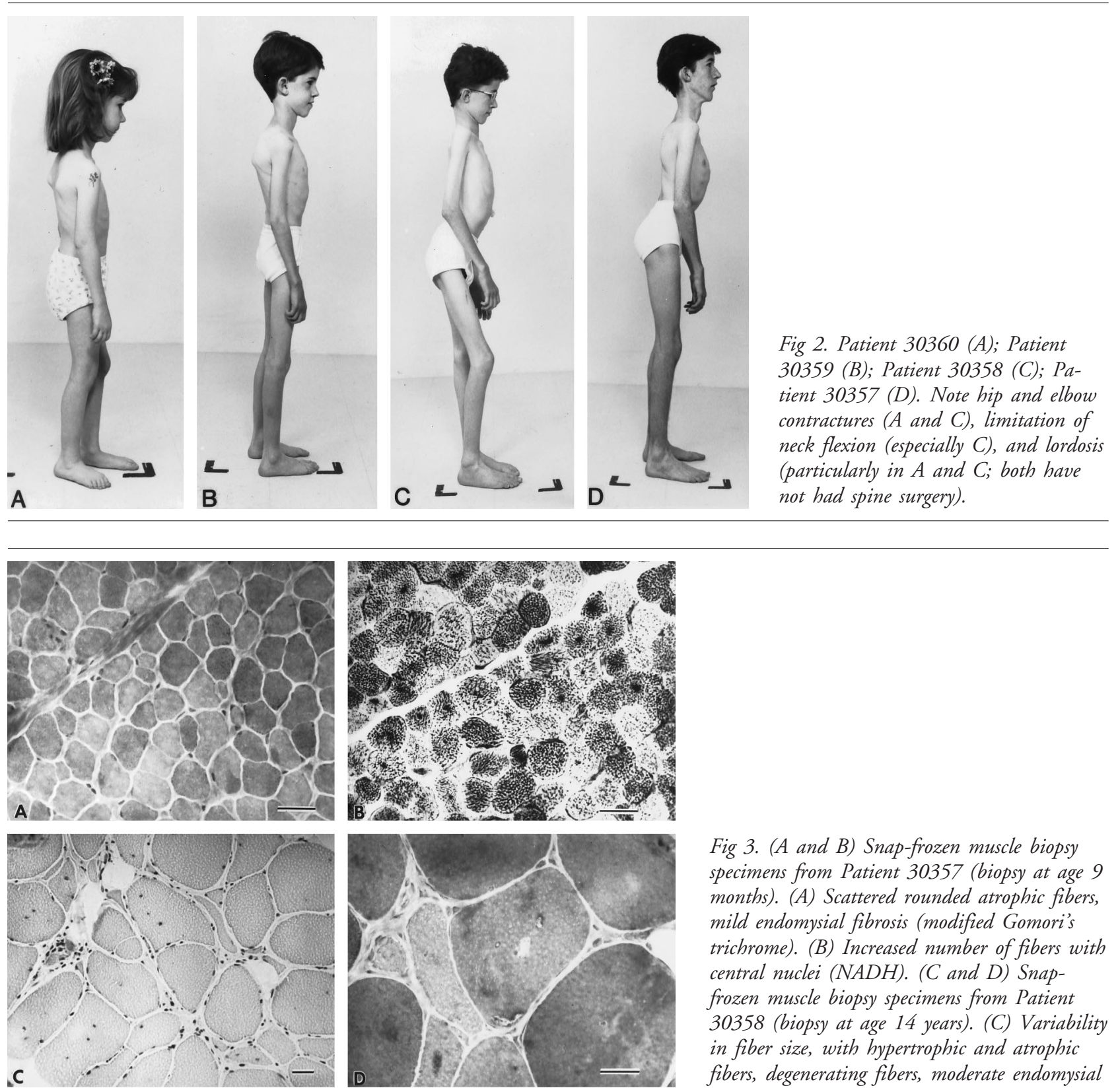

Fig 3. (A and B) Snap-frozen muscle biopsy specimens from Patient 30357 (biopsy at age 9 months). (A) Scattered rounded atrophic fibers, mild endomysial fibrosis (modified Gomori's trichrome). (B) Increased number of fibers with central nuclei (NADH). (C and D) Snapfrozen muscle biopsy specimens from Patient 30358 (biopsy at age 14 years). (C) Variability in fiber size, with hypertrophic and atrophic fibers, degenerating fibers, moderate endomysial fibrosis and fatty replacement (hematoxylin and
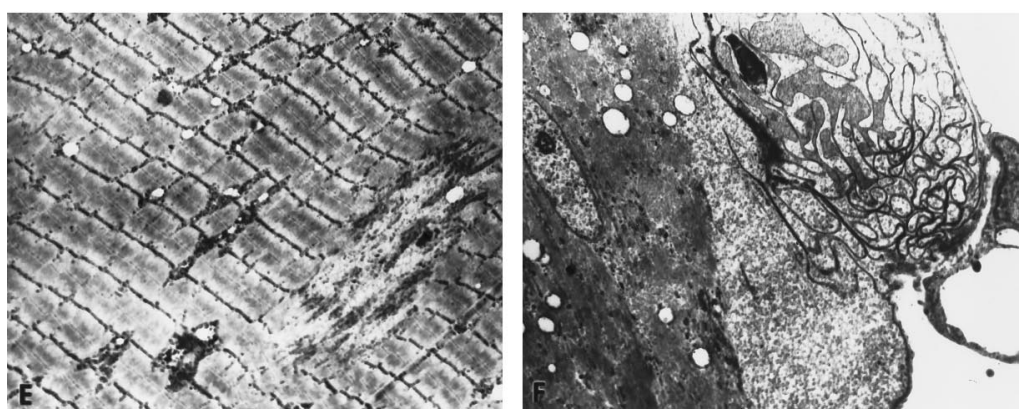
eosin stain). (D) Central and subsarcolemmal vacuoles containing reddish granular material (modified Gomori's trichrome). Bar $=30 \mu \mathrm{m}$ $(A-D)$. (E and F) Electron microscopy (original magnification, $\times 11,400$ before reduction) of muscle biopsy specimens from Patient 30358 (biopsy at age 14 years). (E) Z-band disruption, moderate amount of deposition of glycogen and fat. (F) Subsarcolemmal accumulation of lamellar, myelin-like figures.

15 months, and by 25 months he could raise his arms above his head (although he could not arise from the ground). Because of respiratory insufficiency resulting in several episodes of pneumonia, nocturnal use of a cuirass was instituted at age 8 ; at the time, his forced expiratory vital capacity was measured at $33 \%$ of predicted value, and overnight sleep studies showed oxygen desaturation below 70\%, with episodic hypercarbia at more than $70 \mathrm{~mm} \mathrm{Hg}$. Scoliosis was also 
noted by age 8 ; after wearing a brace for 1 year, he underwent Harrington rod placement at age 10 years.

Examination presently reveals lordosis, minimal elbow contractures, and limited mobility of the neck in flexion and rotation (see Figs 1 and 2). There is minimal eye closure weakness. Neck extensors are stronger (4 of 5) than neck flexors (3 of 5). The limb muscles are markedly atrophic. Strength is symmetric: 4 of 5 in the arms, $4+$ of 5 in the forearms, 3 of 5 in hip flexion, $4+$ of 5 in knee flexion, and 4 of 5 in the distal legs (except the tibialis anterior, graded $4-$ of 5). Reflexes are absent except at the knees, where trace reflexes can be seen with reinforcement. A recent electrocardiogram (ECG) revealed borderline right ventricular conduction delay diagnosed by atypical bimodal R-R' complexes in lead $V_{1}$, with QRS duration in an equivocal range (116 $\mathrm{msec}$ ). A recent echocardiogram revealed normal left ventricular size and function.

PATIENT 30358. A brother, Patient 30358 (see Figs 1 and 2C), is now 14 years old. At his birth, his mother thought him to be unaffected; his symptoms were first noted around 6 months of age, at which time he could sit unsupported but had poor head control. He never crawled, but walked by 18 months of age. Examination at 2.5 years of age revealed weakness of neck extensors and flexors, with limb strength graded at $4-$ of 5 in proximal groups and $4+$ of 5 in distal groups. He arose from the floor with a partial Gower's maneuver, and walked with a lordotic, slightly waddling gait. Reflexes were symmetric but decreased except at the ankles, where they were absent, and toes were downgoing. At the age of 5 years, he was hospitalized with pneumonia and began nocturnal use of a cuirass with supplemental oxygen; at the time, his forced expiratory vital capacity was $34 \%$ of predicted value, and an overnight oximetry study revealed episodes of oxygen desaturation of less than $85 \%$, with hypercarbia of more than $80 \mathrm{~mm} \mathrm{Hg}$. Nasogastric tube feeding was begun at age 5 to maintain body weight; a gastrostomy tube was later placed, and strength minimally but noticeably improved over the next years. At age 9 years, his nocturnal ventilatory support was changed to bilevel positive airway pressure ventilation. At age 12 years, he was noted to have scoliosis and increasing proximal leg weakness associated with increasing hip and hamstring contractures, with knee extension limited to $45^{\circ}$ bilaterally (see Fig 2). At age 13 years, he underwent a hip contracture release, at which time a biopsy of the right quadriceps was performed (see pathology section and Fig 3C-F).

Examination presently reveals scoliosis, lordosis, and contractures of the elbows, knees, and hips. There is limited mobility of the neck in flexion and rotation, as well as severe limitation in spine flexion; the thoracic spine is, in essence, spontaneously fused (see Figs 1 and 2). Neck extensors are stronger ( 4 of 5 ) than neck flexors ( 3 of 5 ). The limb muscles are markedly atrophic. Strength is symmetric: 4 of 5 in the deltoids, $4-$ of 5 in the triceps and biceps, 4 of 5 in the forearms; 2 of 5 in hip flexion, $4+$ of 5 in knee extension, 4 of 5 in the tibialis anterior, $4+$ of 5 gastrocnemius, 2 of 5 in the extensor hallucis, and 0 of 5 in the extensor digitorum brevis. (He is the weakest of the siblings.) Reflexes are absent. A recent ECG revealed borderline right ventricular con- duction delay, diagnosed by atypical bimodal R-R' complexes in lead $V_{1}$, with QRS duration in an equivocal range (94 msec). No echocardiogram has been performed.

PATIENT 30359. Another brother, Patient 30359 (see Figs 1 and $2 \mathrm{~B}$ ), now age 11 years, was noted to have hypotonia and poor head control soon after birth. He sat unsupported at age 6 months and walked by age 18 months, at which time he was noted to have mild proximal weakness and poor head control. He developed respiratory insufficiency with nocturnal $\mathrm{CO}_{2}$ retention, requiring nocturnal cuirass use by age 2 years. Scoliosis developed by age 6 years, and he underwent Harrington rod placement at age 7 . Bilateral hamstring contractures were noted by age 9 .

Examination presently reveals lordosis and moderate elbow contractures as well as limited mobility of the neck in flexion and rotation. Neck extensor and flexor strength are equal ( 4 of 5). Limb muscles are atrophic, but bulk is preserved relative to his older brothers. Strength is 4 of 5 and symmetric except at the triceps (4- of 5) and quadriceps (4+ of 5). Reflexes are absent. A recent ECG is normal, as is a recent echocardiogram (left ventricular ejection fraction $=$ $66 \%)$. A recent forced expiratory vital capacity measurement was $26 \%$ of predicted value.

PATIENT 30360. A sister, Patient 30360 (see Figs 1, and 2A and C), is now 7 years old. A few months after her birth, her mother suspected she might be affected because she was "a little bit floppy." By the age of 18 months she was noted to have poor head control, with a minimal head lag when pulled to a sitting position. She was also noted to have shoulder hypotonia and mild muscle weakness but was considered less affected than her brothers were at a similar age; she reached more motor milestones (running in a stable fashion, which her brothers could not do). She developed scoliosis requiring bracing by age 4, at which age she was noted to use a partial Gower's maneuver and to have marked pronation of the feet during stance and gait. She has been prone to respiratory infections but has not required nocturnal ventilatory support. No ECG or echocardiogram has been performed. Overnight oxygen saturation oximetry alone has been performed, with a median value of $92 \%$, with transient desaturations to $60 \%$.

Examination presently reveals scoliosis and joint laxity in the legs and arm. There is minimal neck and spinal flexion restriction; she cannot touch her chin to her chest and only minimally curves her thoracic spine in flexion (touching her toes). Strength of neck flexion is 3 of 5 and neck extension 4 of 5 . Strength is $4-$ of 5 in the arms and 4 of 5 in the forearms; knee flexion is $4+$ of 5 and knee extension is 5 of 5 ; and strength in the distal legs is 5 of 5 . The only asymmetry is seen in hip flexion, which is 3 of 5 on the right and $4-$ of 5 on the left. Reflexes are absent.

\section{Methods}

IMMUNOHISTOCHEMICAL STUDIES. Immunohistochemical studies were performed as described previously, ${ }^{22}$ using the following primary antibodies: monoclonal antibodies against dystrophin C-terminus (dy8/6C5; 1:100), $\beta$-dystroglycan (43DAG/8D5; 1:100), laminin- $\alpha 2$ C-terminus (Mer3/22B2; 
1:500) all from Novocastra Laboratories, Newcastle upon Tyne, UK; monoclonal antibodies against $\alpha$-sarcoglycan (Ad1/20A6; 1:50); $\beta$-sarcoglycan (BSarc1/23D6; 1:100), $\gamma$-sarcoglycan (35DAG/21B5; 1:100), and $\delta$-sarcoglycan (dSarc3/12C1; 1:100), produced in collaboration with L. V. B. Anderson (Newcastle upon Tyne, UK) monoclonal antibody against caveolin-3 (Transduction Labs, Lexington, $\mathrm{KY}$; 1:1,000); rat monoclonal antibody $4 \mathrm{H} 8-2$, directed against the laminin- $\alpha 2 \mathrm{~N}$-terminus (kind gift from Lydia Sorokin; 1:2); an affinity-purified polyclonal rabbit (rabbit 235) antibody produced against sarcospan (1:50); and a polyclonal rabbit antibody against $\alpha 7 \mathrm{~B}$-integrin (kind gift from Ulrike Mayer; 1:200).

MAGNETIC RESONANCE IMAGING. Imaging was performed on a 1.5-T scanner, version 5.7 EchoSpeed (General Electric Medical Systems, Milwaukee, WI). Axial images of both thighs were obtained simultaneously, using contiguous $10-\mathrm{mm}$ sections in either the quadrature head coil or torso phased array (Medical Advances, Milwaukee, WI) with the following scan parameters: (1) T1-weighted spin echo with a repetition time (TR) between 566 and $800 \mathrm{msec}$, echo time (TE) of $16 \mathrm{msec}, 1$ excitation, a field of view of 22 to $24 \mathrm{~cm}$, and matrix of $256 \times 256$. Imaging time was less than 4 minutes to reduce motion artifact. (2) Short tau inversion recovery fast spin echo (STIR FSE) with a TR between 5,000 and $5,783 \mathrm{msec}$, TE of $52 \mathrm{msec}$, inversion time (TI) of $150 \mathrm{msec}, 8$ echo train, 1 excitation, with a similar field of view, matrix, and slice location as the T1-weighted images.

Muscle signal was inspected on the T1 images for increased signal based on atrophy and fatty replacement. STIR FSE images were inspected for increased signal intensity, which can be typical for acute denervation, or for slightly reduced signal, because of chronic atrophy in the setting of increased fat content. ${ }^{23}$

GENOTYPING. DNA was extracted from peripheral blood by phenol/chloroform techniques followed by isopropanol precipitation. For the purposes of the genomewide screen, DNA was evaluated by polymerase chain reaction with 192 fluorescently labeled primers selected from the Utah Development Group index linkage mapping set (composed of 347 highly polymorphic markers with an average distance of 9.2 $\mathrm{cM}$ across the human genome $\left.{ }^{24}\right)$. By using these primers, genomic DNA was amplified in reaction volumes of $20 \mu \mathrm{l}$ in an MJR PTC-200 thermocycler. Genotypes were visualized on an Applied Biosystems model 373A and evaluated by using the Genotyper peak-calling software.

For evaluation of markers at the RSMD1 locus, genomic DNA was amplified in reaction volumes of $25 \mu \mathrm{l}$ in an MJR PTC-200 thermocycler. End-labeling of primers and amplification of genomic DNA was performed as previously described, ${ }^{25}$ except that reaction times were as follows: (1) 1 cycle at $94^{\circ} \mathrm{C}$ for 4 minutes; (2) 5 cycles, each at $94^{\circ} \mathrm{C}$ for 10 seconds, $58^{\circ} \mathrm{C}$ for 10 seconds, $72^{\circ} \mathrm{C}$ for 20 seconds; (3) 30 cycles, each at $94^{\circ} \mathrm{C}$ for 10 seconds, $55^{\circ} \mathrm{C}$ for 10 seconds, $72^{\circ} \mathrm{C}$ for 20 seconds; (4) $72^{\circ} \mathrm{C}$ for 7 minutes; and $(5) 4^{\circ} \mathrm{C}$ soak. The products were electrophoresed through $5 \%$ denaturing polyacrylamide gels and visualized by autoradiography.
LINKAGE ANALYSIS. Linkage was run on the MLINK routine of the FASTLINK linkage analysis package, using an autosomal recessive inheritance model. Disease allele frequency was set at 0.001 , and $95 \%$ penetrance was assumed. Simulations for the optimal expected LOD score were performed by using the same model and assuming a four-allele system with equal allele frequencies.

\section{Results \\ Pathology}

Patient 30357 (see Fig 2D) had a muscle biopsy performed in a vastus medialis muscle at age 9 months. This reveals scattered rounded atrophic fibers, with a minimal amount of endomysial fibrosis (see Fig 3A). No necrotic, degenerating, or regenerating fibers are seen. Rare areas contain an increased frequency of central nuclei (see Fig 3B), but these are present in very few of the fibers. Fiber cytoarchitecture is normal, including under oil immersion $(100 \times$ lens); specifically, no cores (central or multiple/minicores) or nemaline rods are seen. Staining with ATPase at ph 9.4 reveals a normal mosaic pattern, without fiber-type disproportion or predominance.

Patient 30358 (see Fig 2C) underwent quadriceps muscle biopsy as part of a hip contracture release at the age of 14 years, but the muscle biopsied was not specified. This specimen reveals variation in myofiber size, with rounded atrophic and hypertrophic fibers (see Fig 3C). Scattered degenerating, split, and flocculated fibers are present, but no frankly necrotic fibers are present. Moderate endomysial fibrosis and fatty replacement are present. Fiber cytoarchitecture is generally normal, including under oil immersion $(100 \times$ lens); specifically, no cores (central or multiple/minicores) or nemaline rods are seen. However, a single otherwise normal fiber contains central and subsarcolemmal vacuoles containing reddish granular material on modified Gomori's trichrome stain (see Fig 3D). Staining with ATPase at $\mathrm{pH} 9.4$ reveals a normal mosaic pattern, without fiber-type disproportion or predominance. Electron microscopy reveals areas of Z-band disruption, with moderately increased amounts of glycogen and fatty infiltration (see Fig 3E). An area of subsarcolemmal accumulation of lamellar, myelin-like figures is seen (see Fig 3F), presumably representing autophagic vacuoles. Immunofluorescent studies, using primary antibodies directed to dystrophin, proteins of the dystrophin-glycoprotein complex, caveolin-3, laminin-2 (merosin), and $\alpha 7 \mathrm{~B}$-integrin, are normal.

\section{Radiology}

MRI scanning of the thighs was performed in each child. In all patients, the primary changes were noted on the T1-weighted images consisting of volume loss and elevated signal (Fig 4). The STIR FSE images demonstrated no evidence of increased signal intensity. 

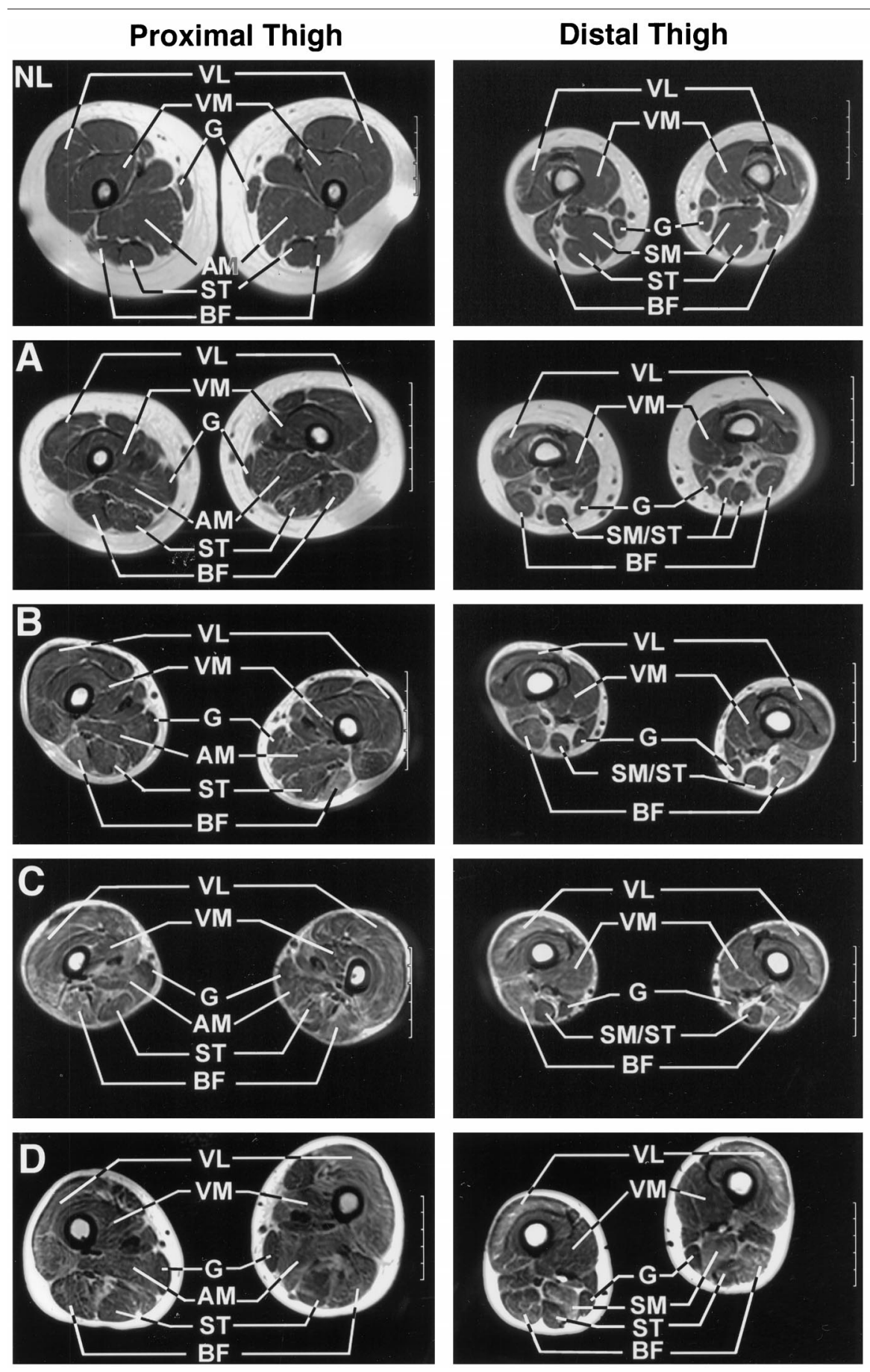

Fig 4. T1-weighted magnetic resonance imaging of the proximal and distal thigh. NL, normal 7-year-old girl (age- and sex-matched control to Patient 30360). Patient 30360 (A); Patient 30359 (B); Patient 30358 (C); Patient 30357 (D). VL = vastus lateralis; $\mathrm{VM}=$ vastus medialis; $\mathrm{G}=$ gracilis; $\mathrm{AM}=$ adductor magnus; $\mathrm{ST}=$ semitendinous; $\mathrm{SM}=$ semimembranous; $\mathrm{BF}=$ biceps femoris. Hatched size marker represents 5 $\mathrm{cm}$ marked at 1-cm intervals.

As judged by both the severity of the T1 signal change and by the number of muscles with signal abnormalities, the degree of muscle involvement varies proportionately to the age of the patient. In the youngest child (see Fig 4A), specific muscles have increased signal (particularly the vastus lateralis and the biceps femoris), whereas adjacent or nearby muscles have normal signals (particularly the vastus medialis and the semitendinous, as well as the adductor magnus and graci- lis). The same pattern of selective muscle involvement is seen in each child.

\section{Genetic Studies}

Initially, we performed a genomewide screen for regions of homozygosity, based on the hypothesis that unknown consanguinity existed within the family (because of the proximity of ancestors, on adjacent farms, about 150 years ago). Only one of these screening 
markers (UT5412 on chromosome 8) was both fully informative and demonstrated that all 4 affected children were homozygous for the same allele. Two nearby markers, D8S1721 and D8S520, were within 0 to 2 cM of UT5412 (as identified by the CEPH database "close markers" function [http://www.cephb.fr/cgi-bin/ $\mathrm{wdb} / \mathrm{ceph} /$ systeme/form]) and were fully informative; neither identified a region of homozygosity around UT5412, challenging that UT5412 is contained within a region homozygous by descent.

Because no regions of homozygosity were defined, the hypothesis of remote consanguinity was abandoned. Linkage analysis was performed, using the 192 genotypes from the homozygosity screen. In this family, the maximum simulated two-point LOD score for fully informative markers is 1.81 at $\Theta=0.00$, representing the maximum statistical power in this family, given its structure (using the model described in $\mathrm{Pa}$ tients and Methods). Linkage analysis of markers from the screening set gave the same LOD score markers at only two loci. One such locus was at UT5412 on chromosome 8 , and the other locus included the adjacent screening markers UT6136 and UT551 (separated by $10 \mathrm{cM}$ on chromosome 12). No muscle diseases have been linked to these regions of chromosome 8 and 12 .

Because of the phenotypic similarity between our patients and those recently reported with CMD-RSS, ${ }^{20}$ we next genotyped markers across the RSMD1 locus on chromosome $1 \mathrm{p} 35-36$. This locus was previously defined by the flanking markers D1S2864 and D1S2794. ${ }^{20}$ The two-point LOD score for the fully informative markers within the RSMD1 locus, D1S2674 and D1S2885, is 1.81 at $\Theta=0.00$ (Table), equal to the maximum simulated LOD score. Genotypes at this locus reveal parental haplotypes, which segregate with the disease trait; all
4 children inherit the same haplotypes from their parents (Fig 5). An obligate recombination event in Patient 30360 defines the telomeric limit of the haplotype at marker D1S458. The centromeric flanking marker is defined by recombination events at the marker D1S200 in 2 individuals; whether the recombination events have occurred in individuals 30357 and 30358 or (alternatively) in individuals 30359 and 30360 cannot be determined. In our family, the flanking markers D1S458 and D1S200 are the limits of a haplotype spanning a locus of $26.5 \mathrm{cM}$, which overlaps the previously reported lo$\mathrm{cus}^{20}$; the RSMD1 locus is, thus, narrowed to $3.0 \mathrm{cM}$ (Fig 6).

\section{Discussion}

\section{Clinical Features}

These 4 siblings affected by CMD-RSS demonstrate a stereotyped clinical course marked by the following features: (1) infantile hypotonia, with prominent neck muscle weakness and poor head control in the early years of life; (2) initial improvement in muscle strength which parallels development, followed by stabilization or only minimal decrease in muscle strength, with marked loss of muscle bulk; (3) contractures of the spine, resulting in rigidity in flexion and, to a lesser extent, contractures of limb joints; (4) skeletal abnormalities, particularly scoliosis, which appear by age 5 years, and may progress to require surgical intervention; (5) early respiratory insufficiency, with onset before adolescence, often requiring nocturnal ventilatory support;, (6) normal intellectual function; and (7) essentially normal cardiac function (excepting mild cardiac conduction defects, diagnosed not by strict but by borderline criteria).

Table. Two-Point LOD Scores for Markers at the RSMD1 Locus

\begin{tabular}{|c|c|c|c|c|c|c|c|c|}
\hline \multirow[b]{2}{*}{ Marker } & \multicolumn{8}{|c|}{ Recombination Distance $(\theta)$} \\
\hline & 0.0 & 0.001 & 0.01 & 0.05 & 0.1 & 0.2 & 0.3 & 0.4 \\
\hline D1S2864 & $-\infty$ & -1.18 & -0.022 & 0.35 & 0.49 & 0.46 & 0.29 & 0.09 \\
\hline$D 1 S 2698$ & $-\infty$ & -1.18 & -0.022 & 0.35 & 0.49 & 0.46 & 0.29 & 0.09 \\
\hline D1S482 & 0.90 & 0.90 & 0.89 & 0.81 & 0.72 & 0.52 & 0.30 & 0.09 \\
\hline$D 1 S 458$ & $-\infty$ & -1.18 & -0.022 & 0.35 & 0.49 & 0.46 & 0.29 & 0.09 \\
\hline D1S2674 & 1.81 & 1.80 & 1.77 & 1.63 & 1.44 & 1.03 & 0.60 & 0.19 \\
\hline D1S234 & 0.90 & 0.90 & 0.89 & 0.81 & 0.72 & 0.52 & 0.30 & 0.09 \\
\hline D1S2885 & 1.81 & 1.80 & 1.77 & 1.63 & 1.44 & 1.03 & 0.60 & 0.19 \\
\hline D1S2749 & 0.90 & 0.90 & 0.89 & 0.81 & 0.72 & 0.52 & 0.30 & 0.09 \\
\hline$D 1 S 470$ & 1.81 & 1.80 & 1.77 & 1.63 & 1.44 & 1.03 & 0.60 & 0.19 \\
\hline D1S2787 & 0.90 & 0.90 & 0.89 & 0.81 & 0.72 & 0.52 & 0.30 & 0.09 \\
\hline D1S449 & 0.90 & 0.90 & 0.89 & 0.81 & 0.72 & 0.52 & 0.30 & 0.09 \\
\hline D1S511 & 0.90 & 0.90 & 0.89 & 0.81 & 0.72 & 0.52 & 0.30 & 0.09 \\
\hline D1S233 & 0.90 & 0.90 & 0.89 & 0.81 & 0.72 & 0.52 & 0.30 & 0.09 \\
\hline D1S193 & 1.81 & 1.80 & 1.77 & 1.63 & 1.44 & 1.03 & 0.60 & 0.19 \\
\hline D1S200 & $-\infty$ & -3.38 & -2.71 & -1.44 & -0.89 & -0.39 & -0.15 & -0.04 \\
\hline
\end{tabular}

Italicized markers are fully informative in this family. Markers in boldface fall within the narrowed locus (see text and Fig 6). 


\begin{tabular}{|c|c|c|c|c|c|c|c|c|c|}
\hline & \multicolumn{4}{|c|}{30355} & \multicolumn{3}{|c|}{30356} & & \\
\hline D1S2864 & & & 23 & 3 & & 1 & 4 & & \\
\hline D1S2698 & & & \begin{tabular}{l|l}
2 & 1
\end{tabular} & 1 & & 1 & 2 & & \\
\hline D1S482 & & & \begin{tabular}{l|l}
3 & 2
\end{tabular} & 2 & & 1 & 1 & & \\
\hline D1S458 & & & \begin{tabular}{l|l}
3 & 2
\end{tabular} & 2 & & 3 & 1 & & \\
\hline D1S2674 & & & 32 & 2 & & 1 & 2 & & \\
\hline D1S234 & & & 12 & 2 & & 3 & 3 & & \\
\hline D1S2885 & & & 13 & 3 & & 3 & 2 & & \\
\hline D1S2794 & & & 11 & 1 & & 4 & 3 & & \\
\hline D1S470 & & & 12 & 2 & & 3 & 4 & & \\
\hline D1S2787 & & & 22 & 2 & & 1 & 2 & & \\
\hline D1S449 & & & 22 & 2 & & 2 & 1 & & \\
\hline D1S511 & & & 22 & 2 & & 2 & 1 & & \\
\hline D1S233 & & & 22 & 2 & & 2 & 1 & & \\
\hline D1S193 & & & 21 & 1 & & 4\lfloor & 3 & & \\
\hline D1S200 & & & 22 & 2 & & 2 & 1 & & \\
\hline & & & & & & & & & \\
\hline D1S2864 & 2 & 4 & 2 & 4 & 2 & 4. & & 2 & 1. \\
\hline D1S2698 & 2 & 2 & 2 & 2 & 2 & 2 & & 2 & 1 \\
\hline D1S482 & 3 & 1 & 3 & 1 & 3 & 1 & & 3 & 1 \\
\hline D1S458 & 3 & 1 & 3 & 1 & 3 & 1 & & 3 & 3 \\
\hline D1S2674 & 3 & 2 & 3 & 2 & 3. & 2 & & 3 & 2 \\
\hline D1S234 & 1 & 3 & 1 & 3 & 1 & 3 & & 1 & 3 \\
\hline D1S2885 & 1 & 2 & 1. & 2 & 1 & 2 & & 1 & 2. \\
\hline D1S2749 & 1 & 3 & 1 & 3 & 1 & 3 & & 1 & 3 \\
\hline D $1 S 470$ & 1 & 4 & 1 & 4 & 1 & 4 & & 1 & 4 \\
\hline D1S2787 & 2 & 2 & 2 & 2 & 2 & 2 & & 2 & 2 \\
\hline D1S449 & 2 & 1 & 2 & 1 & 2 & 1 & & 2 & 1 \\
\hline D1S511 & 2 & 1 & 2 & 1 & 2 & 1 & & 2 & 1 \\
\hline D1S233 & 2 & 1 & 2 & 1 & 2 & 1 & & 2 & 1 \\
\hline D1S193 & 2 & 3 & 2 & 3 & 2 & 3 & & \begin{tabular}{|l|} 
\\
\end{tabular} & 3 \\
\hline D1S200 & 2 & 1 & 2 & 1 & 2 & 2 & & 22 & 2 \\
\hline
\end{tabular}

Fig 5. Genotypes at the chromosome 1p RSMD1 locus. Dashed lines show the limits of the RSMD1 locus as described by Moghadaszadeh and colleagues. ${ }^{20}$ Bold arrow marks the obligate recombination event that narrows the locus. Light arrow points to the other flanking recombination site. Disease haplotypes in our family are outlined by boxes. Markers in bold fall within the narrowed locus as defined in text (also see Fig 6).

Each of these clinical features is, in isolation, nonspecific. Spinal rigidity is a prominent feature of Emery-Dreifuss muscular dystrophy, in both the $\mathrm{X}$-linked and autosomal forms, ${ }^{13,14,26}$ and is also seen in several forms of congenital myopathy. ${ }^{15,18,27}$ In a similar manner, respiratory insufficiency is a nonspecific feature of congenital myopathies, where it may be underrecognized. ${ }^{28}$ However, when these features are present, as in our family, in a stereotyped fashion, and particularly with the striking pattern of selective muscle involvement, they can be recognized as constituting a distinct syndrome.

The familial syndrome we describe is more clinically

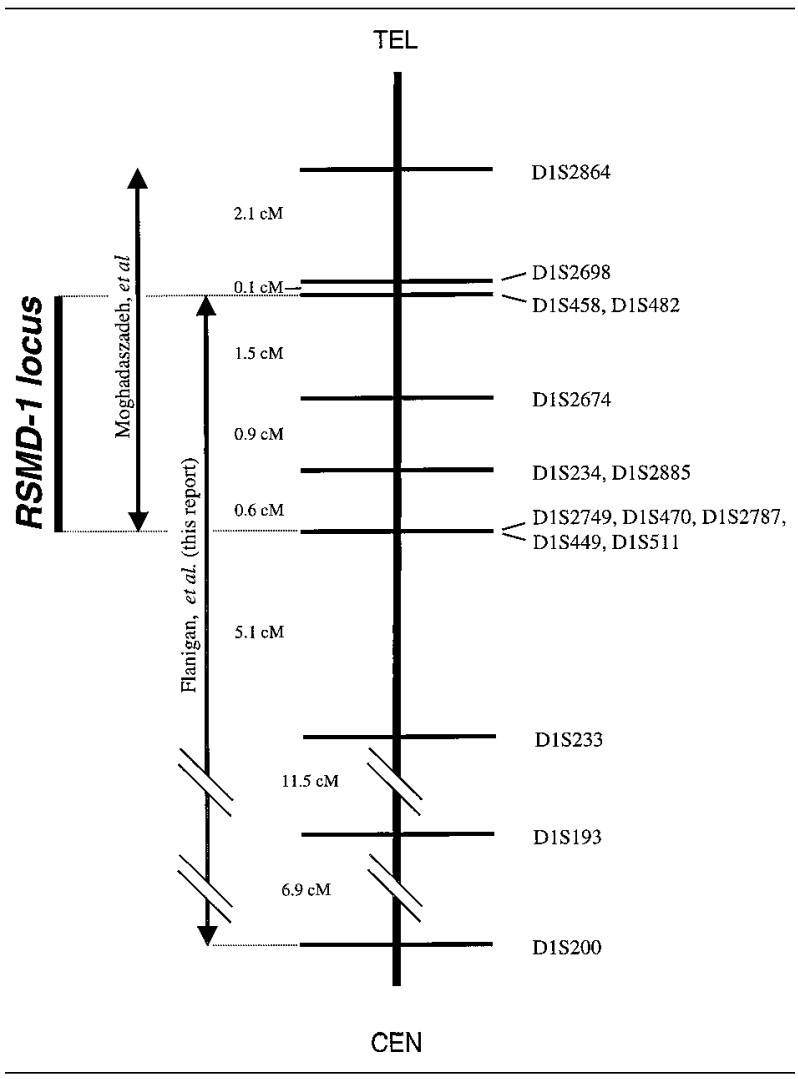

Fig 6. The RSMD1 locus. The haplotypes in our family narrow the gene locus to $3 \mathrm{cM}$, flanked by the markers D1S458 and D1S2749.

homogeneous within this single family than the syndrome previously described in 5 patients from three families from Morocco, Turkey, and Iran. ${ }^{20}$ In the 4 siblings from the family we report, symptoms were evident by the age of 6 months in each child (at birth in 2 ), and all children walked by the age of 18 months. Among the 3 siblings from a single family in the previous report, age of symptom onset ranged from birth to 1 year, and 1 sibling never walked (by the age of 4 years). ${ }^{20}$ In our family, scoliosis is a early feature with ages of onset at 4, 6, 8, and 12 years. In the previous report, scoliosis was noted at a later age; none of the 3 children younger than age 7 years had scoliosis, and the 2 older children developed scoliosis at the ages of 10 and 11 years. ${ }^{20}$ Despite these minor phenotypic differences, the core features of the syndrome are identical.

\section{Pathological and Radiological Features}

Pathologically, the distinction between the CMDs and the congenital myopathies is a somewhat arbitrary one, related to the severity of the changes seen on muscle biopsy. The congenital myopathies are a heterogeneous group of disorders marked by hypotonia and weakness, usually clinically obvious from birth or early childhood. Most forms are associated with specific features 
evident on histopathological or ultrastructural examination of muscle biopsies; examples include nemaline body myopathy, centronuclear myopathy, and central core myopathy. ${ }^{29}$ In contrast, CMD is typically marked by severe and chronic myopathic changes, including endomysial fibrosis and myofiber degeneration. ${ }^{1}$

It is possible that the discordance in histopathology between the two biopsy specimens that we describe may be caused by progression of disease from infancy (when Patient 30357 was biopsied) to adolescence (when Patient 30358 was biopsied). This possibility would be hard to prove without studying sequential muscle biopsies from the same muscle in the same patient over time. We favor an alternative explanation, supported by the stereotyped pattern of signal abnormalities seen on MRI in each child - that is, that muscle involvement is selective during the course of disease. Because the precise muscle biopsied in Patient 30358 during his hip contracture release was not specified, we cannot correlate MRI findings to pathology in this patient, to test this hypothesis. Whether this pattern of signal abnormality is specific to CMD-RSS is unclear, as muscle MRI scanning is not commonly used in evaluation of congenital myopathies and dystrophies.

Because the pathological changes on biopsy of $\mathrm{Pa}$ tient 30357 (at age 9 months) were so mild and nonspecific, these children were followed for years under the rubric of minimal change myopathy, a term first proposed by Dubowitz ${ }^{30}$ to describe, in analogy to minimal change nephropathy, congenital myopathy without specific pathological changes on muscle biopsy. He described several cases characterized by infantile hypotonia and weakness, followed by a fairly stable course of slowly progressive muscle weakness and leading to respiratory insufficiency. These cases did not otherwise have distinguishing clinical features, and were defined by the absence of pathological features diagnostic of other congenital myopathies. In at least one of Dubowitz's cases, severe myopathic changes were found in selective muscles early in the disease at a time when nearby muscles had minimal and nonspecific changes, suggesting that muscle imaging may maximize the yield of a myopathic diagnosis. ${ }^{31}$ The similarities between some cases reported as minimal change myopathy and our CMD-RSS patients, particularly regarding selective muscle involvement when evaluated by biopsy, suggest that these earlier reports may describe patients with disease linked to the RSMD1 locus. Furthermore, they suggest that all children with stereotypic features undergo muscle imaging studies to direct the choice of biopsy site, particularly if a radiologically undirected biopsy reveals only minimal myopathic change. Finally, they emphasize the need for careful examination of spinal flexion in all patients with myo- pathic syndromes who have nonspecific, minimal changes on muscle biopsy.

\section{Genetics}

The two-point LOD scores for four fully informative markers at the RSMD1 locus represent the maximum possible LOD scores for this family structure (see $\mathrm{Ta}$ ble). Although LOD scores for this small family alone are less than those generally accepted as significant evidence in favor of linkage (LOD, >3.0), combining the LOD score in our family with those of the previously reported families, ${ }^{20}$ who share an essentially identical phenotype, results in a summated LOD score of 6.29 for the most informative marker at this locus (D1S2885). Although identical LOD scores are found at two other loci on chromosomes 8 and 12, no muscular dystrophy syndromes have been mapped to those loci. Furthermore, given that a LOD score of 1.81 correlates to an odds ratio of 64:1 against chance association of allele distribution and the disease trait, the presence of such a LOD score at two of 191 independent loci is not unexpected. Assuming that this family's syndrome is linked to $R S M D 1$, an obligate recombinant event (see Fig 5) between the disease and marker D1S458 is observed that defines the telomeric border of the RSMD1 locus. In combination with the previous report, this recombination narrows the RSMD1 locus from $6 \mathrm{cM}$ to a $3 \mathrm{cM}$ region flanked by the markers D1S458 and D1S2794 (see Fig 6).

Two candidate genes have previously been noted to map in or near the RSMD1 locus, ${ }^{20}$ genes that encode microfibrillary-associated protein 2 and heparan sulfate; both of these proteins are constituents of the extracellular matrix and may conceivably be involved in a muscular dystrophy (particularly given the role of laminin-2, an extracellular matrix protein, in another form of CMD). However, these map outside of the narrowed locus according to radiation hybrid data (http://www. ncbi.nlm.nih.gov/genemap/). Other candidate genes might include nuclear envelope proteins, as both the $\mathrm{X}$-linked and autosomal dominant forms of EmeryDreifuss muscular dystrophy, which share spinal rigidity as a common feature with CMD-RSS, are the result of mutations in genes encoding proteins that localize to the nuclear envelope. ${ }^{13,26}$

\section{Summary}

CMD-RSS represents a distinct subtype of CMD in which genetic heterogeneity exists. ${ }^{20} \mathrm{~A}$ core set of symptoms and signs define the syndrome linked to the RSMD1 locus, including congenital or infantile hypotonia and stable or slowly progressive weakness, poor head control, early spinal rigidity, and early scoliosis; these apparently are expressed in a stereotyped fashion within affected families. Early respiratory insufficiency accounts for most life-threatening complications, and 
attention to nocturnal hypoventilation is necessary. Identification of additional families linked to the RSMD1 locus will facilitate the identification of the disease gene. Diagnosis may be aided by attention to the degree of spinal rigidity and by consideration of MRI scanning of thigh muscles before biopsy.

We thank Dr Victor Dubowitz for helpful discussions; Linda Ballard and Adrianne Walker, of the University of Utah Genotyping Core Facility; Brad Nelson and Julian Maack, of the University of Utah Department of Medical Illustrations, for photography (B.N.) and assistance in composition of figures (J.M.); Diana Lim for assistance in composition of figures; Henry Buswell, of the University of Utah Department of Radiology; Shari Brunner for reference assistance; and the family described for their cooperation.

\section{References}

1. Dubowitz V. Muscle disorders in childhood. London: WB Saunders, 1995

2. Echenne B. Congenital muscular dystrophies. In: Lane RJM, ed. Handbook of neuromuscular disease. New York: Marcel Dekker, 1996:139-150

3. Philpot J, Sewry C, Pennock J, Dubowitz V. Clinical phenotype in congenital muscular dystrophy: correlation with expression of merosin in skeletal muscle. Neuromusc Disord 1995;5: 301-305

4. Helbling Leclerc A, Zhang X, et al. Mutations in the laminin alpha 2-chain gene (LAMA2) causemerosin-deficient congenital muscular dystrophy. Nat Genet 1995;11:216-218

5. Dubowitz V. 50th ENMC International Workshop on Congenital Muscular Dystrophy, 28 February to 2 March 1997, Naarden, The Netherlands. Neuromusc Disord 1997;7:539547

6. Kobayashi K, Nakahori Y, Miyake M, et al. An ancient retrotransposal insertion causes Fukuyama-type congenital muscular dystrophy. Nature 1998;394:388-392

7. Cormand B, Avela K, Pihko $\mathrm{H}$, et al. Assignment of the muscle-eye-brain disease gene to $1 \mathrm{p} 32-\mathrm{p} 34$ by linkage analysis and homozygosity mapping. Am J Hum Genet 1999;64:126135

8. Haltia M, Leivo I, Somer H, et al. Muscle-eye-brain disease: a neuropathological study. Ann Neurol 1997;41:173-180

9. Dubowitz V. Pseudo-muscular dystrophy. Proceedings of the Third Symposium: Research Committee of the Muscular Dystrophy Group of Great Britain. London: Pitman Medical, 1965:57-73

10. Dubowitz V. Rigid spine syndrome: a muscle syndrome in search of a name. Proc R Soc Med 1973;66:219-220

11. Morita H, Kondo K, Hoshino K, et al. Rigid spine syndrome with respiratory failure. J Neurol Neurosurg Psychiatry 1990; 53:782-784

12. Ras GJ, van Staden M, Schultz C, et al. Respiratory manifestations of rigid spine syndrome. Am J Respir Crit Care Med 1994; 150:540-546

13. Bonne G, Di Barletta MR, Varnous S, et al. Mutations in the gene encoding lamin $\mathrm{A} / \mathrm{C}$ cause autosomal dominant EmeryDreifuss muscular dystrophy. Nat Genet 1999;21:285-288

14. Taylor J, Sewry CA, Dubowitz V, Muntoni F. Early onset, autosomal recessive muscular dystrophy with Emery-Dreifuss phenotype and normal emerin expression. Neurology 1998;51: $1116-1120$

15. Topaloglu H, Gogus S, Yalaz K, et al. Two siblings with nemaline myopathy presenting with rigid spine syndrome. Neuromusc Disord 1994;4:263-267

16. Reichmann H, Goebel HH, Schneider C, Toyka KV. Familial mixed congenital myopathy with rigid spine phenotype. Muscle Nerve 1997;20:411-417

17. van Munster ET, Joosten EM, van Munster Uijtdehaage MA, et al. The rigid spine syndrome. J Neurol Neurosurg Psychiatry 1986;49:1292-1297

18. Merlini L, Granata C, Ballestrazzi A, Marini ML. Rigid spine syndrome and rigid spine sign in myopathies. J Child Neurol 1989;4:274-282

19. Poewe W, Willeit H, Sluga E, Mayr U. The rigid spine syndrome-a myopathy of uncertain nosological position. J Neurol Neurosurg Psychiatry 1985;48:887-893

20. Moghadaszadeh B, Desguerre I, Topaloglu H, et al. Identification of a new locus for a peculiar form of congenital muscular dystrophy with early rigidity of the spine, on chromosome 1p35-36. Am J Hum Genet 1998;62:1439-1445

21. Lander ES, Botstein D. Homozygosity mapping: a way to map human recessive traits with the DNA of inbred children. Science 1987;236:1567-1570

22. Duclos F, Broux O, Bourg N, et al. Beta-sarcoglycan: genomic analysis and identification of a novel missense mutation in the LGMD2E Amish isolate. Neuromusc Disord 1998;8:30-38

23. West GA, Haynor DR, Goodkin R, et al. Magnetic resonance imaging signal changes in denervated muscles after peripheral nerve injury. Neurosurgery 1994;35:1077-1085

24. Utah Marker Development Group. A collection of ordered tetranucleotide-repeat markers from the human genome. Am J Hum Genet 1995;57:619-628

25. Flanigan K, Gardner K, Alderson K, et al. Autosomal dominant spinocerebellar ataxia with sensory axonal neuropathy (SCA4): clinical description and genetic localization to chromosome 16q22.1. Am J Hum Genet 1996;59:392-399

26. Bione S, Maestrini E, Rivella $S$, et al. Identification of a novel $\mathrm{X}$-linked gene responsible for Emery-Dreifuss muscular dystrophy. Nat Genet 1994;8:323-327

27. Merlini L, Granata C. Rigid spine syndrome. In: Lane RJM, ed. Handbook of neuromuscular disease. New York: Marcel Dekker, 1996

28. Khan Y, Heckmatt JZ, Dubowitz V. Sleep studies and supportive ventilatory treatment in patients with congenital muscle disorders. Arch Dis Child 1996;74:195-200

29. Goebel HH. Congenital myopathies. Semin Pediatr Neurol 1996;3:152-161

30. Dubowitz V. In: Muscle disorders in childhood. London: WB Saunders, 1978:105-107

31. Heckmatt JZ, Dubowitz V. Ultrasound imaging and directed needle biopsy in the diagnosis of selective involvement in muscle disease. J Child Neurol 1987;2:205-213 